\title{
Towards an Independent Determination of Muon $g-2$ from Muonium Spectroscopy
}

\author{
Cédric Delaunay $\oplus^{1,{ }^{*}}$ Ben Ohayon $\odot^{2, \dagger}$ and Yotam Soreq $\oplus^{3, *}$ \\ ${ }^{1}$ Laboratoire d'Annecy-le-Vieux de Physique Théorique LAPTh, CNRS-USMB, BP 110 Annecy-le-Vieux, F-74941 Annecy, France \\ ${ }^{2}$ Institute for Particle Physics and Astrophysics, ETH Zürich, CH-8093 Zürich, Switzerland \\ ${ }^{3}$ Physics Department, Technion-Israel Institute of Technology, Haifa 3200003, Israel
}

(Received 28 July 2021; accepted 15 November 2021; published 15 December 2021)

\begin{abstract}
We show that muonium spectroscopy in the coming years can reach a precision high enough to determine the anomalous magnetic moment of the muon below one part per million (ppm). Such an independent determination of muon $g-2$ would certainly shed light on the $\sim 2$ ppm difference currently observed between spin-precession measurements and ( $R$-ratio based) standard model predictions. The magnetic dipole interaction between electrons and (anti)muons bound in muonium gives rise to a hyperfine splitting (HFS) of the ground state which is sensitive to the muon anomalous magnetic moment. A direct comparison of the muonium frequency measurements of the HFS at J-PARC and the 1S-2S transition at PSI with theory predictions will allow us to extract muon $g-2$ with high precision. Improving the accuracy of QED calculations of these transitions by about 1 order of magnitude is also required. Moreover, the good agreement between theory and experiment for the electron $g-2$ indicates that new physics interactions are unlikely to affect muonium spectroscopy down to the envisaged precision.
\end{abstract}

DOI: 10.1103/PhysRevLett.127.251801

Introduction.-The long and winding road that leads to the discovery of physics beyond the standard model (SM) may well go through loops. The magnetic moments of the electron and the muon

$$
\vec{\mu}_{\ell}=g_{\ell}\left(\frac{q_{\ell}}{2 m_{\ell}}\right) \vec{S}_{\ell}
$$

$(\ell=e, \mu)$ are shining examples. Indeed, quantum fluctuations in the vacuum of all known and unknown fields inevitably causes their $g_{\ell}$ factors to deviate from the prediction of the Dirac equation by an "anomalous" part $a_{\ell} \equiv\left(g_{\ell}-2\right) / 2$ [1]. Therefore, any accurate measurement of the latter, together with equally accurate theoretical predictions, open the door to new physics (NP) phenomena.

The Muon $g-2$ Collaboration at the Fermi National Accelerator Laboratory (FNAL) recently determined [2-4] the muon anomalous magnetic moment to 460 parts-perbillion ( $\mathrm{ppb}$ ) from spin-precession measurements. The central value is in good agreement with an earlier measurement [5] at the Brookhaven National Laboratory (BNL), leading to a new experimental world average [2]

$$
a_{\mu}^{\exp }=116592061(41) \times 10^{-11},
$$

to $0.35 \mathrm{ppm}$.

Published by the American Physical Society under the terms of the Creative Commons Attribution 4.0 International license. Further distribution of this work must maintain attribution to the author(s) and the published article's title, journal citation, and DOI. Funded by SCOAP ${ }^{3}$.
The SM prediction for $a_{\mu}$ includes contributions from electromagnetic [6-25], strong and weak interactions involving virtual exchange of photons, leptons, hadrons, and $W, Z$ and Higgs bosons. Its evaluation was recently reviewed by the international theory community, finding [26]

$$
a_{\mu}^{\mathrm{SM}}=116591810(43) \times 10^{-11},
$$

where the theory uncertainty is dominated by uncertainties in the (nonperturbative) hadronic vacuum polarization (HVP) and hadronic light-by-light (HLbL) contributions. This result is 4.2 standard deviation smaller than the experimental average, suggesting a possible contribution from NP with a magnitude of

$$
\Delta a_{\mu} \equiv a_{\mu}^{\exp }-a_{\mu}^{\mathrm{SM}}=(251 \pm 59) \times 10^{-11},
$$

which is about $2 \mathrm{ppm}$. The SM prediction in Eq. (3) relies on a leading-order (LO) HVP contribution of $a_{\mu}^{\mathrm{HVP}-\mathrm{LO}}\left(e^{+} e^{-}\right)=6931(40) \times 10^{-11}[10-15,26]$ derived from experimental $e^{+} e^{-} \rightarrow$ hadrons data (the so-called $R$-ratio method) at low-energy using dispersion relations [27-29].

An alternative determination of the LO-HVP contribution stems from an ab initio calculation in lattice QCD [30]. The latest result from the Budapest-Marseille-Wuppertal Collaboration $(\mathrm{BMWc}) a_{\mu}^{\mathrm{HVP}-\mathrm{LO}}($ lattice $)=7075(55) \times$ $10^{-11}$ [31], consistent with previous calculations [32-36] but with a threefold reduced uncertainty, is about $1 \mathrm{ppm}$ larger than the $R$-ratio value and agrees with the experimental average in Eq. (2) within 2 standard deviations. 
The present status of muon $g-2$ is therefore puzzling. Imagining that the discrepancy holds either between experiment and a converged theory (currently $2 \mathrm{ppm}$ ), or between the two theoretical calculations (currently $1 \mathrm{ppm}$ ), then an independent determination of $a_{\mu}$ to this level, demanding as it would be, is of immense importance.

An alternative strategy for determining $a_{\mu}$ would be probing magnetic properties of muons bound in atoms instead of free particles. Muonium $\left(M \equiv \mu^{+} e^{-}\right)$is the bound state of an antimuon and an electron. It is a purely leptonic bound state, free of nuclear structure effects usually hampering the theoretical accuracy in ordinary atoms, for which high-order QED calculations are available. In contrast, muonium spectroscopy is currently limited by experimental uncertainties, being mostly of statistical origin [37]. In this Letter, we show that by pushing muonium spectroscopy to its limits, both theoretical and experimental, a determination of $a_{\mu}$ is possible with $\mathcal{O}(1 \mathrm{ppm})$ precision. This completely different approach would shed a new light on the current puzzle.

Muon $g-2$ from muonium spectroscopy.-One of the most precisely measured spectral line in muonium is the hyperfine splitting (HFS) of its $1 \mathrm{~S}$ ground state $\nu_{\mathrm{HFS}}$ at $12 \mathrm{ppb}$ [38]. The HFS originates from the interaction of the electron spin with the magnetic field sourced by the magnetic dipole moment of the (static) antimuon. For electronic states without orbital momentum, the LO Hamiltonian is a Fermi contact interaction between the electron and muon magnetic moments [39]

$$
H_{\mathrm{HFS}}=-\frac{2 \mu_{0}}{3} \vec{\mu}_{e} \cdot \vec{\mu}_{\mu} \delta^{3}(r),
$$

where $\mu_{0}=2 \alpha h /\left(e^{2} c\right)$ is the vacuum permeability. As it involves explicitly the magnetic moment of the muon, it is in principle sensitive to its anomalous part.

Besides the fine-structure constant $\alpha$, and the Rydberg constant $R_{\infty}=\alpha^{2} m_{e} c /(2 h)$, two parameters are endemic to muonic physics at low energy: the electron-muon mass ratio $m_{e} / m_{\mu}$ and the muon magnetic moment. Hydrogen spectroscopy determines $R_{\infty}$ at 1.9 parts-per-trillion (ppt) [40], making it the most accurately known constant in physics [41]. Combining $R_{\infty}$ with accurate measurements of the electron and rubidium atomic masses and the $h / m_{\mathrm{Rb}}$ ratio gives the currently best determination of $\alpha$ at $81 \mathrm{ppt}$ $[43,44]$. The muonic constants must be extracted from (at least) two other independent observables. $m_{e} / m_{\mu}$ is known at $19 \mathrm{ppb}$ from comparing the measured ground-state HFS of muonium [38] with the SM prediction [47]. Since we envisage to use this observable to extract $a_{\mu}, m_{e} / m_{\mu}$ must be obtained by other means. The current second best determination of the electron-muon mass ratio is at $120 \mathrm{ppb}$, coming from a measurement of the (total) muon magnetic moment [38]. However, this cannot be used either as it clearly depends on $a_{\mu}$. Another way to extract $m_{e} / m_{\mu}$ is to measure a muonium line that is (mostly) independent of the magnetic moment. To date, the only possibility is the Lyman- $\alpha$ line between the $1 \mathrm{~S}$ and $2 \mathrm{~S}$ states.

The theory prediction for the $1 \mathrm{~S}-2 \mathrm{~S}$ transition frequency in muonium is

$$
\nu_{1 \mathrm{~S}-2 \mathrm{~S}}=\frac{3}{4} \frac{R_{\infty} c}{\left(1+m_{e} / m_{\mu}\right)}\left[1+\delta_{1 \mathrm{~S}-2 \mathrm{~S}}\right],
$$

where the muon mass enters as a recoil contribution of $\sim 0.5 \%$ and subleading corrections in $\delta_{1 \mathrm{~S}-2 \mathrm{~S}} \sim \mathcal{O}\left(\alpha^{2}\right)$ are known up to three-loop QED with $20 \mathrm{kHz}$ uncertainty [48].

For the ground-state HFS the theory prediction reads

$$
\nu_{\mathrm{HFS}}=\frac{16}{3}\left(1+a_{\mu}\right) \frac{m_{e}}{m_{\mu}} \frac{R_{\infty} c \alpha^{2}}{\left(1+m_{e} / m_{\mu}\right)^{3}}\left[1+\delta_{\mathrm{HFS}}\right],
$$

where $\delta_{\mathrm{HFS}} \sim \mathcal{O}(\alpha / \pi)$ gathers corrections [49] beyond the Hamiltonian in Eq. (5) from relativistic, radiative (including the anomalous magnetic moment $a_{e}$ of the electron), recoil, radiative-recoil, weak, and hadronic contributions. The theory uncertainty $\delta_{\mathrm{HFS}}$ is about $70 \mathrm{~Hz}[47,50]$, dominated by unknown three-loop QED contributions to the radiative-recoil term in $\delta_{\mathrm{HFS}}$ [47].

Since the $\delta_{\mathrm{HFS}, 1 \mathrm{~S}-2 \mathrm{~S}}$ corrections above only weakly depend on fundamental constants, the leading contributions to $\nu_{\mathrm{HFS}}$ and $\nu_{1 \mathrm{~S}-2 \mathrm{~S}}$ are sufficient to estimate the sensitivity of muonium spectroscopy to muon $g-2$. Combining Eqs. (6) and (7) allows us to separately determine $m_{e} / m_{\mu}$ and $a_{\mu}$, providing a simple estimate of their uncertainties

$$
\begin{gathered}
u^{2}\left(m_{e} / m_{\mu}\right) \simeq u_{r}^{2}\left(R_{\infty}\right)+u_{r}^{2}\left(\nu_{1 \mathrm{~S}-2 \mathrm{~S}}\right) \\
+\delta_{1 \mathrm{~S}-2 \mathrm{~S}}^{2} u_{r}^{2}\left(\delta_{1 \mathrm{~S}-2 \mathrm{~S}}\right), \\
u^{2}\left(a_{\mu}\right) \simeq u_{r}^{2}\left(m_{e} / m_{\mu}\right)+4 u_{r}^{2}(\alpha)+u_{r}^{2}\left(\nu_{\mathrm{HFS}}\right) \\
+\delta_{\mathrm{HFS}}^{2} u_{r}^{2}\left(\delta_{\mathrm{HFS}}\right),
\end{gathered}
$$

where $u_{r}(x) \equiv u(x) / x$ and $u(x)$ is 1 standard deviation of the observable $x$, and $u_{r}\left(\delta_{i}\right)$ denotes the relative theory uncertainty of $\delta_{i}$ that is not associated with the $R_{\infty}, \alpha$, and $m_{e} / m_{\mu}$ parameters.

A least-square adjustment (see Supplemental Material [51], which includes Refs. [52-55]) using 2018 CODATA recommended values for $R_{\infty}$ and $\alpha$, and including the stateof-the-art calculation (see Ref. [40] and references therein) of $\delta_{\mathrm{HFS}, 1 \mathrm{~S}-2 \mathrm{~S}}$ yields

$$
a_{\mu}^{M}=116637(82) \times 10^{-8},
$$

and $m_{e} / m_{\mu}=4836329(4) \times 10^{-9}$, which is larger $\left(a_{\mu}^{M}-a_{\mu}^{\exp } \simeq 4.5 \times 10^{-7}\right)$ but consistent with both the experimental value in Eq. (2) and the theoretical result in Eq. (3). The large uncertainty $u\left(a_{\mu}\right)=8.2 \times 10^{-7}$ is dominated by the experimental $u_{r}\left(\nu_{1 \mathrm{~S}-2 \mathrm{~S}}\right)=4.0 \times 10^{-9}$ [48]. Note that this determination of $a_{\mu}$ assumes that muonium theory follows SM predictions. As shown below, contributions beyond the SM ones related to an 
hypothetical NP coupling to electrons are sufficiently constrained not to affect the least-square adjustment.

Expected improvements in muonium physics.-The present data are lacking precision to provide a competitive determination of muon $g-2$. However the situation is expected to dramatically improve in the near future thanks to new experimental techniques and more accurate QED calculations. We summarize the developments planned at the next round of experiments at PSI and J-PARC and show that, together with the ongoing theory improvement, they will allow for an extraction of $a_{\mu}$ at few ppm. Moreover, we outline and argue on the feasibility of the refinements necessary to bring $u_{r}\left(a_{\mu}\right)$ below the ppm level.

1S-2S transition: The first necessary ingredient is to improve the electron-muon mass ratio from muonium 1S$2 \mathrm{~S}$ spectroscopy. Spectroscopy of the $1 \mathrm{~S}-2 \mathrm{~S}$ transition in hydrogenic atoms relies on two-photon excitation with a UV laser, operating at a $244 \mathrm{~nm}$ wavelength for muonium. The very high transition frequency makes possible measurements at ppt precision, with a $145 \mathrm{kHz}$ natural linewidth due to the muon lifetime.

One of the main challenges is the low excitation efficiency, as the $1 \mathrm{~S}-2 \mathrm{~S}$ transition is a two-photon transition. In order to increase the transition probability, previous $1 \mathrm{~S}-2 \mathrm{~S}$ measurements utilized a high-power pulsed laser. This, however, came at the cost of broadening the linewidth to $20 \mathrm{MHz}$ associated with the laser pulse width. Another main systematic uncertainty of $10 \mathrm{MHz}$ also originated from the pulsed interaction. A high-power pulsed laser changes its frequency during every pulse, an effect known as chirping, which is notoriously difficult to compensate for.

To circumvent the limitations of pulsed laser excitation, the MuoniuM 1Aser SpectroScopy (Mu-MASS) experiment [56] at PSI utilizes a cavity-enhanced continuous wave (cw) excitation [57]. The reduced excitation efficiency in cw operation is compensated by the use of the low-energy-muon (LEM) beam line [58] paired with new methods to obtain slow muonium atoms emitted into vacuum after production in mesoporous thin $\mathrm{SiO}_{2}$ films [59]. With such techniques the Mu-MASS uncertainty goal was set to $10 \mathrm{kHz}$ (4 ppt) [56].

On the theory side the $\nu_{1 \mathrm{~S}-2 \mathrm{~S}}$ uncertainty was quoted at $20 \mathrm{kHz}$ in Ref. [48]. To the best of our knowledge this figure has not been updated, despite the recent improvement in QED calculations for hydrogenlike atoms [60,61]. By rescaling the theory uncertainties for hydrogen [40] to the muon mass (discarding nuclear finite-size and polarizability contributions) we assess the current theory uncertainty of $\nu_{1 \mathrm{~S}-2 \mathrm{~S}}$ to be at $14 \mathrm{kHz}(5.7 \mathrm{ppt})$.

The Rydberg constant is also expected to improve in the next few years, anticipating a full resolution of the so-called proton radius puzzle [62]. The very precise determination of the proton radius from muonic hydrogen [63,64] improves the theoretical precision in hydrogen by about
1 order of magnitude, which then becomes limited by bound-state QED calculations. The current QED uncertainty in hydrogen is roughly $1 \mathrm{kHz}$ [60], allowing, in principle, a threefold [65] more precise determination of $R_{\infty}$ relative to the latest CODATA [40].

Using Eq. (9) the above values (referred to as "ongoing" in Tables I and II) yield an expected precision on $m_{e} / m_{\mu}$ at $1.5 \mathrm{ppb}$.

Ground state HFS: The major improvement to the electron-muon mass ratio considered above opens up the possibility to obtain a value for $a_{\mu}$ with few ppm uncertainty, comparable to the current difference in Eq. (4), granted that the ground state HFS is improved as well. The current best measurement of $\nu_{\mathrm{HFS}}$ was done with a chopped beam at LAMPF and limited by statistics [38]. The Muonium Spectroscopy Experiment Using Microwave (MuSEUM) experiment will improve the statistical uncertainty by using the high-intensity pulsed muon beam at J-PARC $[69,70]$ as well as Rabi-oscillation spectroscopy [71]. Moreover, recently a thorough optimization of the microwave cavity has been done to drive down the systematic uncertainties to the ppb level [68]. This will allow for more precise HFS measurements by about 1 order of magnitude [68] compared to previous LAMPF measurements. We take $10 \mathrm{~Hz}(2.2 \mathrm{ppb})$ as an estimate for the uncertainty goal of the MuSEUM experiment, which is compatible with the "several ppb" in Ref. [68]. (See also Refs. [72,73] and references therein.) Another important systematic uncertainty is due to the pressure shift from the finite gas density environment [74] in which HFS measurement are performed. This can be overcome by measuring the HFS in vacuum using a low-energy muon beam.

TABLE I. Uncertainty budget for the $1 \mathrm{~S}-2 \mathrm{~S}$ transition (in ppt) and 1S HFS (in ppb) in muonium. The first column summarizes the current status of the relative uncertainties $u_{r}(x)=u(x) / x$. The second one (ongoing) indicates the milestone set by the MuMASS and MuSEUM experiments. The last one presents the foreseeable improvements necessary for a muonium determination of $a_{\mu}$ at sub-ppm level. Blank entries correspond to quantities that do not need further improvement.

\begin{tabular}{lccccc}
\hline \hline & & \multicolumn{3}{c}{$u_{r}$} & \\
\cline { 3 - 5 }$\nu_{i}$ (unit) & Quantity & Current & Ongoing & Ultimate & Refs. \\
\hline & QED & 8.1 & 5.7 & 0.7 & {$[48,60]$} \\
& HVP & $\mathcal{O}\left(10^{-2}\right)$ & & & {$[66]$} \\
1S-2S (ppt) & $R_{\infty}$ & 1.9 & 0.65 & & {$[40,60]$} \\
& $\alpha$ & $\mathcal{O}\left(10^{-3}\right)$ & & & {$[40]$} \\
& exp & $3.99 \times 10^{3}$ & 4.1 & 1.6 & {$[48,56]$} \\
& QED & 16 & 2.2 & 0.2 & {$[50,67]$} \\
& HVP & 0.33 & 0.18 & & {$[15,40]$} \\
HFS (ppb) & $\alpha$ & 0.30 & 0.16 & & {$[40,43]$} \\
& $R_{\infty}$ & $\mathcal{O}\left(10^{-3}\right)$ & & & {$[40]$} \\
& $\exp$ & 12 & 2.2 & 0.90 & {$[38,68]$} \\
\hline \hline
\end{tabular}


TABLE II. Uncertainty budget for the determination of $m_{e} / m_{\mu}$ and $a_{\mu}$ from precision muonium spectroscopy. Same as Table I.

\begin{tabular}{lcccc}
\hline \hline & & \multicolumn{3}{c}{$u_{r}$} \\
\cline { 3 - 5 } Parameter (unit) & Quantity & Current & Ongoing & Ultimate \\
\hline \multirow{3}{*}{$m_{e} / m_{\mu}(\mathrm{ppb})$} & $\nu_{1 \mathrm{~S}-2 \mathrm{~S}(\mathrm{exp})}$ & 825 & 0.84 & 0.34 \\
& QED(1S-2S) & 1.7 & 1.2 & 0.1 \\
& $R_{\infty}$ & 0.40 & 0.13 & \\
& total & 825 & 1.5 & 0.37 \\
& $\nu_{1 \mathrm{~S}-2 \mathrm{~S}(\mathrm{exp})}$ & 708 & 0.73 & 0.29 \\
& $\nu_{\mathrm{HFS}}(\mathrm{exp})$ & 10 & 1.9 & 0.77 \\
& $\mathrm{QED}(1 \mathrm{~S}-2 \mathrm{~S})$ & 1.4 & 1.0 & 0.07 \\
$a_{\mu}(\mathrm{ppm})$ & $\mathrm{QED}(\mathrm{HFS})$ & 14 & 1.9 & 0.2 \\
& $\mathrm{HVP}(\mathrm{HFS})$ & 0.29 & 0.16 & \\
& $R_{\infty}$ & 0.35 & 0.13 & \\
& $\alpha$ & 0.26 & 0.14 & \\
& total & 708 & 3.0 & 0.88 \\
\hline \hline
\end{tabular}

Excluding the muon mass uncertainty, the theoretical ground state HFS calculation is currently limited by boundstate QED to around $70 \mathrm{~Hz}$ accuracy [47] coming from uncalculated sets of three-loop diagrams. Efforts to improve this calculation are ongoing, quoting a goal of $10 \mathrm{~Hz}[50]$.

Collecting the theoretical and experimental values discussed above we estimate with Eq. (9) the expected uncertainty for extracting $a_{\mu}$ from the ongoing effort in muonium spectroscopy as about $3 \mathrm{ppm}$ (shown in blue on Fig. 1). Such rousing prospects, comparable to the

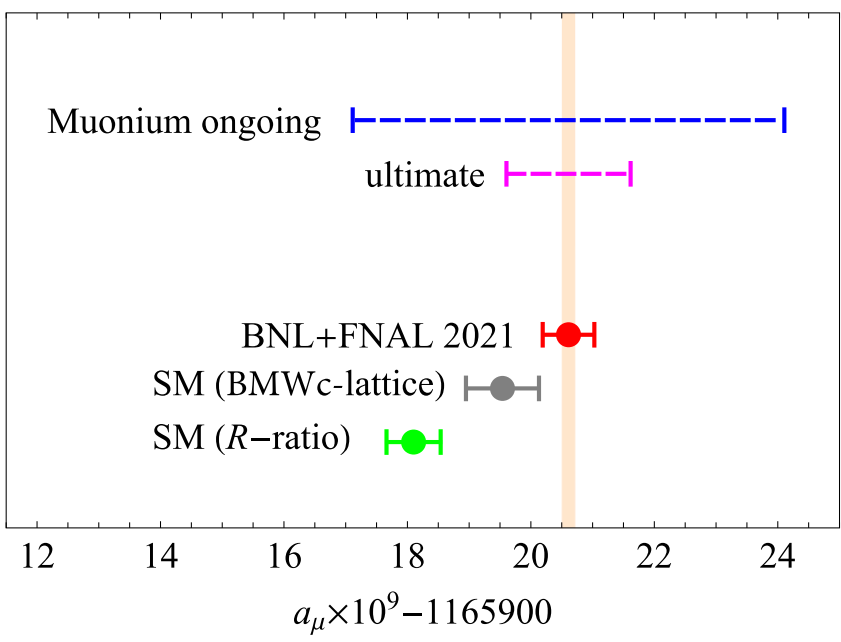

FIG. 1. Anomalous magnetic moment of the muon determined from spin-precession measurements at BNL and FNAL (red) [2], standard model calculations with LO HVP evaluated from $e^{+} e^{-} \rightarrow$ hadrons data (green) [26], or lattice QCD (gray) [31] and the projected sensitivities in muonium (dashed) with the currently planned spectroscopy improvements (blue) and its ultimate improvement (magenta), centered around the current experimental average. The orange band shows the fourfold improved FNAL standard deviation expected in the near future. difference in Eq. (4), would already contribute to the muon $g-2$ puzzle.

Further improvements: Next, we explore how much the above uncertainties can be further reduced. We based our estimations on known experimental techniques, arguing that the necessary theoretical improvements can be reasonably achieved along the way.

An improvement on the $1 \mathrm{~S}-2 \mathrm{~S}$ frequency determination from the Mu-MASS goal of $10 \mathrm{kHz}$ to few $\mathrm{kHz}$ is expected [56]. Since final systematic uncertainties are estimated at the $\mathrm{kHz}$ level [56], this precision could be accommodated by an order-of-magnitude increase in statistics. Such an increase could either come from an improved high-energy muon beam rate, as considered in the high-intensity muon beam (HIMB) upgrade [75-77] at PSI, and/or a higher efficiency in muon moderation, as is pursued by the MuCool Collaboration [78-80]. Moreover, another effort in improving the $1 \mathrm{~S}-2 \mathrm{~S}$ measurement is under consideration at J-PARC [73]. A $1 \mathrm{~S}-2 \mathrm{~S}$ measurement at $4 \mathrm{kHz}$ would be sufficient to make the $m_{e} / m_{\mu}$ uncertainty on $\nu_{\mathrm{HFS}}$ subdominant.

Such an improved experimental precision must be supplemented by higher accuracy calculations. Following recent progress in such calculations $[60,61,81]$, the main limitation for the theoretical uncertainty comes from unknown radiative-recoil corrections of $\mathcal{O}\left[\alpha(Z \alpha)^{6}(m / M)\right]$ [60]. This correction is likely to be calculated in the near future since the recent convergence on the proton radius $[62,82-86]$ makes it the limiting factor to reducing the Rydberg constant uncertainty, as well as the deuterium radius deduced from the H-D isotope shift [87]. The pure recoil correction at $\mathcal{O}\left[(Z \alpha)^{6}(m / M)^{2}\right]$ is also required for $\mathrm{kHz}$ accuracy in $M$. It is partially calculated [88] and there is no known obstacle towards a complete result.

Regarding the ground state HFS, a further improvement upon the ongoing efforts, both in experiment and theory, is more demanding. Experimentally the muon-lifetime constraint on the linewidth already poses a major challenge. A measurement at $10 \mathrm{~Hz}$ precision already requires to resolve the line center to $10^{-4}$ of the linewidth, an achievement similar to the recent $2 \mathrm{~S}-4 \mathrm{P}$ frequency measurement in hydrogen [89]. Further improving the precision with better lineshape modeling would constitute a premiere in spectroscopy. A complementary approach consists in converting extremely high statistics into narrower linewidths by postselecting so-called "old muonium" atoms [38,90], namely, muons that have not decayed after several lifetimes. Therefore, despite the challenges, we imagine that HFS measurements could be done at the ppb level $(\simeq 4 \mathrm{~Hz})$.

As regards systematic uncertainty, down to the ppb level the main one is still considered to be the quadratic pressure shift [38], which could be reduced with lower pressures, relying on higher statistics. Another promising option would be to mix gases such as $\mathrm{He}$ and $\mathrm{Kr}$, which have opposite pressure shift contributions [91]. The needed high 
rates could be accommodated by the HIMB upgrade at PSI that will deliver $\sim 10^{10}$ antimuons per second, which is 3 orders of magnitude higher than the cw muon beam used for the HFS measurement at LAMPF. Leveraging the technical advancements by the MuSEUM Collaboration [68,71], together with a 100 day beam time, we conclude that a sub-ppb experimental uncertainty in $\nu_{\mathrm{HFS}}$ could be envisioned.

The corresponding theory prediction to the level of $4 \mathrm{~Hz}$ accuracy is demanding as well. However, as long as the uncertainty from HVP contributions (presently about $0.8 \mathrm{~Hz}$ [15]) is subdominant, completing the required bound-state QED calculations in the same timescale as experimental milestones could be reasonably envisaged. Indeed, ongoing work already set a goal of a few Hz [67], which would suffice to make $\nu_{\mathrm{HFS}}$ limited by experiment.

Assuming that the $1 \mathrm{~S}-2 \mathrm{~S}$ transition and ground state HFS in muonium are pushed to the aforementioned limits would bring down the $a_{\mu}$ uncertainty to $u\left(a_{\mu}\right) \simeq 103 \times 10^{-11}$ $(0.88 \mathrm{ppm})$, which is about half of the current difference between theory and experiment.

Summary and outlook.-We proposed an alternative strategy to extract the anomalous magnetic moment of the muon based on highly precise measurements and theoretical QED calculations in muonium. Current data on the 1S-2S transition and ground state HFS frequencies allows us to determine $a_{\mu}$ at $\sim 700 \mathrm{ppm}$, largely limited by statistics in the $1 \mathrm{~S}-2 \mathrm{~S}$ measurement. A new program, partly motivated by the present muon $g-2$ puzzle, of highly precise muonium spectroscopy and theory will significantly improve the situation in the coming decade, reducing the $a_{\mu}$ uncertainty to about $3 \mathrm{ppm}$, and even below the ppm level at a future stage. As shown in the Supplemental Material [51], which includes Refs. [92-105], this indirect determination which relies on bound-state QED calculation is unlikely to be affected by NP, given present constraints on exotic forces coupled to electrons.

Such an independent value of $a_{\mu}$ cannot be competitive with spin-precession measurements that are expected to reach $\sim 0.1 \mathrm{ppm}$ accuracy in a few years $[2,106]$. However it would most certainly help interpreting the current difference between these measurements and the ( $R$-ratio based) SM prediction, were it to persist over the same timescale. A muonium value of $a_{\mu}$ favoring today's experimental average, along with $\delta_{1 \mathrm{~S}-2 \mathrm{~S}}$ and $\delta_{\mathrm{HFS}}$ consistent with the SM, would strengthen the evidence of NP dominantly coupled to muons (assuming the $R$-ratio determination of the HVP contribution is a faithful figure of the SM prediction). Conversely, a value consistent with the current $R$-ratio prediction would indicate a potential unknown systematic with the BNL/FNAL measurements, or point to a nontrivial NP dynamics. In the latter case, precision measurements of additional muonium lines, like the 2S-2P Lamb shift $[107,108]$ ongoing at PSI, would help discriminating among different possibilities. Either way, this highly motivates to push the frontier of precision muonium physics as much as possible.

We thank Michael Eides and Yevgeny Stadnik for useful discussions and Paolo Crivelli, Michael Eides, Gilad Perez, and, in particular, Klaus Jungmann for valuable comments on the manuscript. B. O. acknowledges support from the European Union's Horizon 2020 research and innovation program under the Marie Skłodowska-Curie Grant Agreement No. 101019414. Y.S. is supported by the United States-Israel Binational Science Foundation (BSF) (NSF-BSF program Grant No. 2018683), by the Israel Science Foundation (Grant No. 482/20), and by the Azrieli foundation. Y. S. is supported by the Taub Family Foundation.

*edric.delaunay@lapth.cnrs.fr

†bohayon@ethz.ch

†soreqy@physics.technion.ac.il

[1] J.S. Schwinger, On quantum electrodynamics and the magnetic moment of the electron, Phys. Rev. 73, 416 (1948).

[2] B. Abi et al., Measurement of the Positive Muon Anomalous Magnetic Moment to 0.46 ppm, Phys. Rev. Lett. 126, 141801 (2021).

[3] T. Albahri et al., Magnetic-field measurement and analysis for the muon $g-2$ experiment at Fermilab, Phys. Rev. A 103, 042208 (2021).

[4] T. Albahri et al., Measurement of the anomalous precession frequency of the muon in the Fermilab muon $g-2$ experiment, Phys. Rev. D 103, 072002 (2021).

[5] G. W. Bennett et al., Final report of the muon E821 anomalous magnetic moment measurement at BNL, Phys. Rev. D 73, 072003 (2006).

[6] T. Aoyama, M. Hayakawa, T. Kinoshita, and M. Nio, Complete Tenth-Order QED Contribution to the Muon $g-2$, Phys. Rev. Lett. 109, 111808 (2012).

[7] T. Aoyama, T. Kinoshita, and M. Nio, Theory of the anomalous magnetic moment of the electron, Atoms 7, 28 (2019).

[8] A. Czarnecki, W. J. Marciano, and A. Vainshtein, Refinements in electroweak contributions to the muon anomalous magnetic moment, Phys. Rev. D 67, 073006 (2003); Erratum, Phys. Rev. D 73, 119901 (2006).

[9] C. Gnendiger, D. Stöckinger, and H. Stöckinger-Kim, The electroweak contributions to $(g-2)_{\mu}$ after the Higgs boson mass measurement, Phys. Rev. D 88, 053005 (2013).

[10] M. Davier, A. Hoecker, B. Malaescu, and Z. Zhang, Reevaluation of the hadronic vacuum polarisation contributions to the standard model predictions of the muon $g-2$ and $\alpha\left(m_{Z}^{2}\right)$ using newest hadronic cross-section data, Eur. Phys. J. C 77, 827 (2017).

[11] A. Keshavarzi, D. Nomura, and T. Teubner, Muon $g-2$ and $\alpha\left(M_{Z}^{2}\right)$ : A new data-based analysis, Phys. Rev. D 97 , 114025 (2018).

[12] G. Colangelo, M. Hoferichter, and P. Stoffer, Two-pion contribution to hadronic vacuum polarization, J. High Energy Phys. 02 (2019) 006. 
[13] M. Hoferichter, B.-L. Hoid, and B. Kubis, Three-pion contribution to hadronic vacuum polarization, J. High Energy Phys. 08 (2019) 137.

[14] M. Davier, A. Hoecker, B. Malaescu, and Z. Zhang, A new evaluation of the hadronic vacuum polarisation contributions to the muon anomalous magnetic moment and to $\boldsymbol{\alpha}\left(\mathbf{m}_{\mathbf{Z}}^{\mathbf{2}}\right)$, Eur. Phys. J. C 80, 241 (2020); Erratum, Eur. Phys. J. C 80, 410 (2020).

[15] A. Keshavarzi, D. Nomura, and T. Teubner, The $g-2$ of charged leptons, $\alpha\left(M_{Z}^{2}\right)$ and the hyperfine splitting of muonium, Phys. Rev. D 101, 014029 (2020).

[16] A. Kurz, T. Liu, P. Marquard, and M. Steinhauser, Hadronic contribution to the muon anomalous magnetic moment to next-to-next-to-leading order, Phys. Lett. B 734, 144 (2014).

[17] K. Melnikov and A. Vainshtein, Hadronic light-by-light scattering contribution to the muon anomalous magnetic moment revisited, Phys. Rev. D 70, 113006 (2004).

[18] P. Masjuan and P. Sánchez-Puertas, Pseudoscalar-pole contribution to the $\left(g_{\mu}-2\right)$ : A rational approach, Phys. Rev. D 95, 054026 (2017).

[19] G. Colangelo, M. Hoferichter, M. Procura, and P. Stoffer, Dispersion relation for hadronic light-by-light scattering: Two-pion contributions, J. High Energy Phys. 04 (2017) 161.

[20] M. Hoferichter, B.-L. Hoid, B. Kubis, S. Leupold, and S. P. Schneider, Dispersion relation for hadronic light-by-light scattering: Pion pole, J. High Energy Phys. 10 (2018) 141.

[21] A. Gérardin, H. B. Meyer, and A. Nyffeler, Lattice calculation of the pion transition form factor with $N_{f}=2+1$ Wilson quarks, Phys. Rev. D 100, 034520 (2019).

[22] J. Bijnens, N. Hermansson-Truedsson, and A. RodríguezSánchez, Short-distance constraints for the HLbL contribution to the muon anomalous magnetic moment, Phys. Lett. B 798, 134994 (2019).

[23] G. Colangelo, F. Hagelstein, M. Hoferichter, L. Laub, and P. Stoffer, Longitudinal short-distance constraints for the hadronic light-by-light contribution to $(g-2)_{\mu}$ with large$N_{c}$ Regge models, J. High Energy Phys. 03 (2020) 101.

[24] T. Blum, N. Christ, M. Hayakawa, T. Izubuchi, L. Jin, C. Jung, and C. Lehner, The Hadronic Light-by-Light Scattering Contribution to the Muon Anomalous Magnetic Moment from Lattice QCD, Phys. Rev. Lett. 124, 132002 (2020).

[25] G. Colangelo, M. Hoferichter, A. Nyffeler, M. Passera, and P. Stoffer, Remarks on higher-order hadronic corrections to the muon $g-2$, Phys. Lett. B 735, 90 (2014).

[26] T. Aoyama et al., The anomalous magnetic moment of the muon in the standard model, Phys. Rep. 887, 1 (2020).

[27] C. Bouchiat and L. Michel, La résonance dans la diffusion méson $\pi$-méson $\pi$ et le moment magnétique anormal du méson $\mu$, J. Phys. Radium 22, 121 (1961).

[28] S. J. Brodsky and E. De Rafael, Suggested boson-lepton pair couplings and the anomalous magnetic moment of the muon, Phys. Rev. 168, 1620 (1968).

[29] M. Gourdin and E. De Rafael, Hadronic contributions to the muon g-factor, Nucl. Phys. B10, 667 (1969).

[30] T. Blum, Lattice Calculation of the Lowest Order Hadronic Contribution to the Muon Anomalous Magnetic Moment, Phys. Rev. Lett. 91, 052001 (2003).
[31] Sz. Borsanyi et al., Leading hadronic contribution to the muon 2 magnetic moment from lattice QCD, Nature (London) 593, 51 (2021).

[32] A. Gérardin, M. Cè, G. von Hippel, B. Hörz, H. B. Meyer, D. Mohler, K. Ottnad, J. Wilhelm, and H. Wittig, The leading hadronic contribution to $(g-2)_{\mu}$ from lattice QCD with $N_{\mathrm{f}}=2+1$ flavours of $\mathrm{O}(a)$ improved Wilson quarks, Phys. Rev. D 100, 014510 (2019).

[33] C. T. H. Davies, C. DeTar, A. X. El-Khadra, E. Gámiz, S. Gottlieb et al., Hadronic-vacuum-polarization contribution to the muon's anomalous magnetic moment from four-flavor lattice QCD, Phys. Rev. D 101, 034512 (2020).

[34] D. Giusti, V. Lubicz, G. Martinelli, F. Sanfilippo, and S. Simula, Electromagnetic and strong isospin-breaking corrections to the muon $g-2$ from lattice QCD + QED, Phys. Rev. D 99, 114502 (2019).

[35] T. Blum, P. A. Boyle, V. Gülpers, T. Izubuchi, L. Jin, C. Jung, A. Jüttner, C. Lehner, A. Portelli, and J. T. Tsang, Calculation of the Hadronic Vacuum Polarization Contribution to the Muon Anomalous Magnetic Moment, Phys. Rev. Lett. 121, 022003 (2018).

[36] Sz. Borsanyi et al., Hadronic Vacuum Polarization Contribution to the Anomalous Magnetic Moments of Leptons from First Principles, Phys. Rev. Lett. 121, 022002 (2018).

[37] K. P. Jungmann, Precision muonium spectroscopy, J. Phys. Soc. Jpn. 85, 091004 (2016).

[38] W. Liu, M. G. Boshier, S. Dhawan, O. van Dyck, P. Egan et al., High Precision Measurements of the Ground State Hyperfine Structure Interval of Muonium and of the Muon Magnetic Moment, Phys. Rev. Lett. 82, 711 (1999).

[39] C. Cohen-Tannoudji, B. Diu, and F. Laloë, Quantum Mechanics, 1st ed. (Wiley, New York, NY, 1977) [Translation of Mécanique Quantique (Hermann, Paris, 1973)].

[40] E. Tiesinga, P. J. Mohr, D. B. Newell, and B. N. Taylor, CODATA recommended values of the fundamental physical constants: 2018, Rev. Mod. Phys. 93, 025010 (2021).

[41] A more recent $1 \mathrm{~S}-3 \mathrm{~S}$ frequency measurement [42] provides another determination of $R_{\infty}$ at $3.5 \mathrm{ppt}$ consistent with the CODATA value.

[42] A. Grinin, A. Matveev, D. C. Yost, L. Maisenbacher, V. Wirthl, R. Pohl, T. W. Hänsch, and T. Udem, Two-photon frequency comb spectroscopy of atomic hydrogen, Science 370, 1061 (2020).

[43] L. Morel, Z. Yao, P. Cladé, and S. Guellati-Khélifa, Determination of the fine-structure constant with an accuracy of 81 parts per trillion, Nature (London) 588, 61 (2020).

[44] This value disagrees with the previous best determination of $\alpha$ [45] by $5.4 \sigma$ [43], possibly due to systematic errors [46]. While puzzling, this discrepancy is too small (relative to uncertainties in muonium) to affect significantly present and future determinations of $a_{\mu}$.

[45] R. H. Parker, C. Yu, W. Zhong, B. Estey, and H. Müller, Measurement of the fine-structure constant as a test of the Standard Model, Science 360, 191 (2018).

[46] H. Müller, Standard model of particle physics tested by the fine-structure constant, Nature (London) 588, 37 (2020).

[47] M. I. Eides, Hyperfine splitting in muonium: Accuracy of the theoretical prediction, Phys. Lett. B 795, 113 (2019). 
[48] V. Meyer, S. N. Bagayev, P. E. G. Baird, P. Bakule, M. G. Boshier et al., Measurement of the 1s-2s Energy Interval in Muonium, Phys. Rev. Lett. 84, 1136 (2000).

[49] P. J. Mohr, D. B. Newell, and B. N. Taylor, CODATA recommended values of the fundamental physical constants: 2014, Rev. Mod. Phys. 88, 035009 (2016).

[50] M. I. Eides and V. A. Shelyuto, Hyperfine splitting in muonium and positronium, Int. J. Mod. Phys. A 31, 1645034 (2016).

[51] See Supplemental Material at http://link.aps.org/ supplemental/10.1103/PhysRevLett.127.251801 for technical details about the least-square adjustment and the details about possible contribution of new physics to the muonium energy levels.

[52] P. J. Mohr and B. N. Taylor, CODATA recommended values of the fundamental physical constants: 1998, Rev. Mod. Phys. 72, 351 (2000).

[53] F. G. Mariam et al., Higher Precision Measurement of the hfs Interval of Muonium and of the Muon Magnetic Moment, Phys. Rev. Lett. 49, 993 (1982).

[54] S. G. Karshenboim and V. A. Shelyuto, Hadronic vacuumpolarization contribution to various QED observables, Eur. Phys. J. D 75, 49 (2021).

[55] M. I. Eides and V. A. Shelyuto, Three-loop contributions to hyperfine splitting: Muon loop light-by-light insertion and other closed lepton loops, Phys. Rev. D 90, 113002 (2014).

[56] P. Crivelli, The Mu-MASS (Muonium laser SpectroScopy) experiment, Hyperfine Interact. 239, 49 (2018).

[57] Z. Burkley, L. de Sousa Borges, B. Ohayon, A. Golovozin, J. Zhang, and P. Crivelli, Stable high power deep-uv enhancement cavity in ultra high vacuum with fluoride coatings, Opt. Express 29, 27450 (2021).

[58] E. Morenzoni, F. Kottmann, D. Maden, B. Matthias, M. Meyberg, Th. Prokscha, Th. Wutzke, and U. Zimmermann, Generation of Very Slow Polarized Positive Muons, Phys. Rev. Lett. 72, 2793 (1994).

[59] A. Antognini, P. Crivelli, T. Prokscha, K. S. Khaw, B. Barbiellini, L. Liszkay, K. Kirch, K. Kwuida, E. Morenzoni, F. M. Piegsa, Z. Salman, and A. Suter, Muonium Emission into Vacuum from Mesoporous Thin Films at Cryogenic Temperatures, Phys. Rev. Lett. 108, 143401 (2012).

[60] S. G. Karshenboim, A. Ozawa, V. A. Shelyuto, R. Szafron, and V.G. Ivanov, The Lamb shift of the $1 s$ state in hydrogen: Two-loop and three-loop contributions, Phys. Lett. B 795, 432 (2019).

[61] V. A. Yerokhin, K. Pachucki, and V. Patkos, Theory of the lamb shift in hydrogen and light hydrogen-like ions, Ann. Phys. (Amsterdam) 531, 1800324 (2019).

[62] J.-P. Karr, D. Marchand, and E. Voutier, The proton size, Nat. Rev. Phys. 2, 601 (2020).

[63] R. Pohl et al., The size of the proton, Nature (London) 466, 213 (2010).

[64] A. Antognini et al., Proton structure from the measurement of $2 S-2 P$ transition frequencies of muonic hydrogen, Science 339, 417 (2013).

[65] M. Horbatsch and E. A. Hessels, Tabulation of the boundstate energies of atomic hydrogen, Phys. Rev. A 93, 022513 (2016).
[66] J. L. Friar, J. Martorell, and D. W. L. Sprung, Hadronic vacuum polarization and the Lamb shift, Phys. Rev. A 59, 4061 (1999).

[67] M. I. Eides, Hyperfine splitting in muonium: Theory meets experiment, in Proceedings of the International Workshop on Physics of Muonium and Related Topics (2018).

[68] K. S. Tanaka, M. Iwasaki, O. Kamigaito, S. Kanda, N. Kawamura, Y. Matsuda, T. Mibe, S. Nishimura, N. Saito, N. Sakamoto, S. Seo, K. Shimomura, P. Strasser, K. Suda, T. Tanaka, H. A. Torii, A. Toyoda, Y. Ueno, and M. Yoshida, Development of microwave cavities for measurement of muonium hyperfine structure at J-PARC, arXiv:2104.06663.

[69] H. A. Torii, H. A. Torii, M. Aoki, Y. Fukao, Y. Higashi, T. Higuchi, H. Iinuma, Y. Ikedo, K. Ishida, M. Iwasaki et al., Precise measurement of muonium hfs at J-PARC muse, in Proceedings of the 2nd International Symposium on Science at J-PARC-Unlocking the Mysteries of Life, Matter and the Universe- (2015), p. 025018, 10.7566/ JPSCP.8.025018.

[70] Y. Ueno et al., New precision measurement of muonium hyperfine structure, Proc. Sci., ICHEP2018 (2019) 466.

[71] S. Nishimura et al., Rabi-oscillation spectroscopy of the hyperfine structure of muonium atoms, Phys. Rev. A 104, 020801 (2021).

[72] T. Asaka, M. Tanaka, K. Tsumura, and M. Yoshimura, Precision electroweak shift of muonium hyperfine splitting, arXiv:1810.05429.

[73] A. Keshavarzi, K. S. Khaw, and T. Yoshioka, Muon $g-2$ : Current status, arXiv:2106.06723.

[74] S. Kanda et al., New precise spectroscopy of the hyperfine structure in muonium with a high-intensity pulsed muon beam, Phys. Lett. B 815, 136154 (2021).

[75] D. Kiselev, P. Baumann, P. Duperrex, S. Jollet, P.-R. Kettle, A. Knecht, D. Laube, C. Nyfeler, A. Papa, D. Reggiani et al., Progress and challenges of the psi meson targets and relevant systems, in Proceedings of the 3rd J-PARC Symposium (J-PARC2019) (2021), p. 011102, 10.7566/ JPSCP.33.011102.

[76] D. Kiselev, C. Baumgarten, R. Dölling, P. Duperrex, D. Götz, J. Grillenberger, D. Reggiani, M. Schneider, M. Schippers, M. Seidel et al., Status and future projects of the psi high intensity proton accelerator, in Proceedings of the 3rd J-PARC Symposium (J-PARC2019) (2021), p. 011004, 10.7566/JPSCP.33.011004.

[77] A. Knecht et al., Science Case for the new High-Intensity Muon Beams HIMB at PSI, arXiv:2111.05788.

[78] I. Belosevic, A. Antognini, Y. Bao, A. Eggenberger, M. Hildebrandt, R. Iwai, D. M. Kaplan, K. S. Khaw, K. Kirch, A. Knecht et al. muCool: A next step towards efficient muon beam compression, Eur. Phys. J. C 79, 430 (2019).

[79] A. Antognini, N. J. Ayres, I. Belosevic, V. Bondar, A. Eggenberger, M. Hildebrandt, R. Iwai, D. M. Kaplan, K. S. Khaw, K. Kirch, A. Knecht, A. Papa, C. Petitjean, T. J. Phillips, F. M. Piegsa, N. Ritjoho, A. Stoykov, D. Taqqu, and G. Wichmann, Demonstration of Muon-Beam Transverse Phase-Space Compression, Phys. Rev. Lett. 125, 164802 (2020). 
[80] A. Antognini and D. Taqqu, muCool: Muon cooling for high-brightness $\mu$ beams, SciPost Phys. Proc. 5, 0030 (2021).

[81] M. I. Eides and V. A. Shelyuto, Three-loop spinindependent radiative-recoil corrections to energy levels in muonium, arXiv:2110.13279.

[82] A. Grinin, A. Matveev, D. C. Yost, L. Maisenbacher, V. Wirthl, R. Pohl, T. W. Hänsch, and T. Udem, Two-photon frequency comb spectroscopy of atomic hydrogen, Science 370, 1061 (2020).

[83] W. Ubachs, Crisis and catharsis in atomic physics, Science 370, 1033 (2020).

[84] N. Bezginov, T. Valdez, M. Horbatsch, A. Marsman, A. C. Vutha, and E. A. Hessels, A measurement of the atomic hydrogen lamb shift and the proton charge radius, Science 365, 1007 (2019).

[85] H.-W. Hammer and U.-G. Meißner, The proton radius: From a puzzle to precision, Sci. Bull. 65, 257 (2020).

[86] Z.-F. Cui, D. Binosi, C. D. Roberts, and S. M. Schmidt, Fresh Extraction of the Proton Charge Radius from Electron Scattering, Phys. Rev. Lett. 127, 092001 (2021).

[87] K. Pachucki, V. Patkóš, and V. A. Yerokhin, Three-photon exchange nuclear structure correction in hydrogenic systems, Phys. Rev. A 97, 062511 (2018).

[88] I. R. Blokland, A. Czarnecki, and K. Melnikov, Expansion of bound state energies in powers of $\mathrm{m} / \mathrm{M}$ and $(1-\mathrm{m} / \mathrm{M})$, Phys. Rev. D 65, 073015 (2002).

[89] A. Beyer et al., The Rydberg constant and proton size from atomic hydrogen, Science 358, 79 (2017).

[90] M. G. Boshier, S. Dhawan, X. Fei, V. W. Hughes, M. Janousch et al., Observation of resonance line narrowing for old muonium, Phys. Rev. A 52, 1948 (1995).

[91] B. K. Rao, D. Ikenberry, and T. P. Das, Hyperfine pressure shift and van der Waals interaction. IV. Hydrogen-rare-gas systems, Phys. Rev. A 2, 1411 (1970).

[92] C. Frugiuele, J. Pérez-Ríos, and C. Peset, Current and future perspectives of positronium and muonium spectroscopy as dark sectors probe, Phys. Rev. D 100, 015010 (2019).

[93] S. G. Karshenboim, Precision Physics of Simple Atoms and Constraints on a Light Boson with Ultraweak Coupling, Phys. Rev. Lett. 104, 220406 (2010).
[94] R. Jackiw and S. Weinberg, Weak interaction corrections to the muon magnetic moment and to muonic atom energy levels, Phys. Rev. D 5, 2396 (1972).

[95] F. Jegerlehner and A. Nyffeler, The muon g-2, Phys. Rep. 477, 1 (2009).

[96] R. Balkin, C. Delaunay, M. Geller, E. Kajomovitz, G. Perez, Y. Shpilman, and Y. Soreq, A custodial symmetry for muon g-2, Phys. Rev. D 104, 053009 (2021).

[97] A. Messiah, Quantum Mechanics (Dover Publications, New York, 2014).

[98] E. E. Salpeter and H. A. Bethe, A relativistic equation for bound state problems, Phys. Rev. 84, 1232 (1951).

[99] D. Hanneke, S. Fogwell, and G. Gabrielse, New Measurement of the Electron Magnetic Moment and the Fine Structure Constant, Phys. Rev. Lett. 100, 120801 (2008).

[100] E. Hardy and R. Lasenby, Stellar cooling bounds on new light particles: Plasma mixing effects, J. High Energy Phys. 02 (2017) 033.

[101] X. Fan and G. Gabrielse, Driven one-particle quantum cyclotron, Phys. Rev. A 103, 022824 (2021).

[102] G. Gabrielse, S. E. Fayer, T. G. Myers, and X. Fan, Towards an improved test of the standard model's most precise prediction, Atoms 7, 45 (2019).

[103] P. Ilten, Y. Soreq, M. Williams, and W. Xue, Serendipity in dark photon searches, J. High Energy Phys. 06 (2018) 004.

[104] Yu. M. Andreev et al., Constraints on New Physics in Electron $g-2$ from a Search for Invisible Decays of a Scalar, Pseudoscalar, Vector, and Axial Vector, Phys. Rev. Lett. 126, 211802 (2021).

[105] C. Delaunay et al. (to be published).

[106] M. Abe et al., A new approach for measuring the muon anomalous magnetic moment and electric dipole moment, Prog. Theor. Exp. Phys. 2019, 053C02 (2019).

[107] G. Janka et al., Intense beam of metastable Muonium, Eur. Phys. J. C 80, 804 (2020).

[108] B. Ohayon, G. Janka, I. Cortinovis, Z. Burkley, L. de Bourges-Sousa, E. Depero, A. Golovizin, X. Ni, Z. Salman, A. Suter et al., Precision measurement of the lamb shift in muonium, arXiv:2108.12891. 